\title{
Type A behavior pattern, multiple-task performance, and subjective estimation of mental workload
}

\author{
DIANE L. DAMOS and KATHRYN A. BLOEM \\ Arizona State University, Tempe, Arizona
}

\begin{abstract}
This paper examines the relation between the Type A behavior pattern, individual differences in multiple-task performance, and the dissociation between performance and subjective estimates of mental workload. Sixteen females completed the Jenkins Activity Survey and performed a variety of information processing tasks under single- and dual-task conditions. After each task, subjects rated the workload they had experienced on eight bipolar adjective scales. The slope of the memory-search task was the only single-task performance measure that showed a significant difference between Type As (36 msec) and Type Bs (68 msec). However, on three of the four dual-task combinations, Type As had faster response times than Type Bs. Dissociations between performance and subjective estimates of workload were apparent between Type A and Type B individuals on the frustration and fatigue scales. Type As reported less frustration and more fatigue under single-task than under dual-task conditions, whereas Type Bs reported the opposite pattern.
\end{abstract}

During the last 100 years, there has been a continual interest in individual differences in multiple-task performance. Most of this interest has centered on the notion of a general time-sharing ability (see Ackerman, Schneider, \& Wickens, 1984, for a review of the most recent studies). Because so much effort has focused on time-sharing abilities, psychologists have failed to investigate other potential sources of these differences in multiple-task performance.

One such source is between-subject differences in the Type A coronary-prone behavior pattern (Friedman \& Rosenman, 1974). Individuals who score high on measures of the Type A pattern are characterized by an extreme sense of time urgency, typically trying to accomplish more and more in less and less time. Additionally, Type A individuals perform better on tasks that have no performance deadlines (Burnam, Pennebaker, \& Glass, 1975) and prefer to work at a more rapid pace than individuals who obtain lower scores (Type B individuals) (Frankenhaeuser, Lundberg, \& Forsman, 1980).

These results suggest that Type A subjects should perform better under multiple-task conditions than Type B subjects because speed is even more critical under multiple-task situations than under single-task. Thus, individuals who set their own deadlines and like to work at a rapid pace should perform better than those who do not have these characteristics. To date, however, only two experiments (Glass et al., 1980; Matthews \& Brunson, 1979) have compared Type A and Type B subjects under

This research was supported by NASA-Ames Cooperative Agreement NCC 2-202. Sandy Hart was the scientific monitor. The authors would like to thank Alfred Guardino for his help wth the data collection. Requests for reprints should be sent to Diane Damos, Department of Psychology, Arizona State University, Tempe, AZ 85287. dual-task conditions, and these studies gave conflicting results.

Although Glass et al. (1980) found no significant difference in performance between Type A and Type B subjects on either task, Type As reported being more nervous and tense than Type Bs. These results hint at the dissociation often found between subjective workload ratings and multiple-task performance (Derrick \& Wickens, 1984), a serious problem that currently restricts the usefulness of subjective estimates. Manuck and Garland (1979) also suggested that the Type A behavior pattern may be involved in such dissociations. In their study, Type As were less satisfied with their performance than Type Bs even though Type As actually performed better than Type Bs.

The study described below was part of a larger experiment designed to examine the relation between multipletask performance, subjective estimates of workload, the Type A behavior pattern, and several information processing measures (Damos, 1984). Only the data relating to the Type A/Type B behavior pattern are discussed here.

\section{METHOD}

\section{Subjects}

Sixteen right-handed female subjects were paid $\$ 22.00$ for participating. Nine of these subjects had scores of 5 or below (mean of 4.0 ) and seven subjects had scores of 13 and above (mean of 14.4) on the Jenkins Activity Survey as revised for college students (Krantz, Glass, \& Snyder, 1974). All were between the ages of 18 and 35 years and were native English speakers. Bonuses were given for good performance on the running-difference matrix and the memory-classification combinations described below.

\section{Apparatus}

A DEC $11 / 23$ recorded all responses and performed the timing for all tasks, except the digit-span task. The subjects made their responses by depressing a key on each of two $4 \times 4$ matrix keyboards mounted 
on the arms of their chairs. All stimuli for these tasks were displayed on a CRT screen. An Apple Ile system with two disk drives and an Amdek monitor were used to present the digit-span task.

\section{Tasks}

Dependent measures. The first six tasks listed below used the same dependent measures: the percentage of correct responses and a response time (RT) measure, which was calculated by dividing the number of correct responses for the trial into $60 \mathrm{sec}$. At the end of each trial, these were displayed to the subject along with the total number of responses. The other tasks used the indicated measures. The first six tasks were unpaced; as soon as a response was made, the stimulus was erased and a new one was presented.

Memory search. Positive set sizes of three, four, or five letters were presented on the display at the beginning of each trial. The presentation of the memory set was followed by a probe digit. If the probe was a member of the positive set, the subject pressed the key under her right index finger. If it was not, she pressed the key under her right middle finger. This task was performed alone and with the time-estimation task.

Choice RT. In this task, randomly selected digits were presented sequentially to the subject. The difficulty of the task was manipulated by varying the number of equally probable stimulus alternatives. In the two-alternative (1-bit) condition, the digits 1 and 2 were used; in the four-alternative (2-bit) condition, the digits 1 through 4 were used; in the eight-alternative (3-bit) condition, the digits 1 through 8 were used. The subject responded by pressing the appropriate key on her right-hand keyboard. This task was performed alone and with the time-estimation task.

Matrix. For this task, $5 \times 5$ matrix grids with five randomly selected illuminated cells were presented sequentially to the subject. The subject's task was to determine whether the current matrix was a rotated version of the preceding matrix. Only $90^{\circ}$ right or $90^{\circ}$ left rotations were permitted. If the current matrix was a rotated version of the preceding matrix, the subject responded "same" by pressing a key under her left index finger. If the current matrix was not a rotated version of the preceding matrix, the subject responded "different" by pressing a key under her left middle finger. The response to the first matrix pattern of any trial was always "same." For every trial, approximately $50 \%$ of the correct responses were "same"' and 50\% "different." A matrix pattern was allowed to repeat itself (in its rotated form) a maximum of four times. This task was performed alone and with the running-difference task.

Running difference. In this task, randomly selected digits between 0 and 8 were presented sequentially to the subject. The subject responded with the absolute difference between the most recently displayed digit and the preceding digit by pressing one of eight keys on her right-hand keyboard. All nine digits were presented with approximately the same frequency, and a digit was never allowed to repeat itself. The digits were presented visually. The middle two horizontal rows of keys were used. The response to the first stimulus of any trial was always " 1 ."

Under dual-task conditions, the matrix was displayed as it was in singletask conditions, and the digits for the running-difference task were displayed below the matrix. The two tasks were completely independent of each other.

Classification. For this task, two randomly selected digits between 5 and 8 were presented simultaneously to the subject. The digits varied on two dimensions: size and name. The subject determined the number of dimensions on which the stimuli were alike and then pressed one of three keys on her left-hand keyboard. This task was performed alone and with the memory task.

Memory. In this task, randomly selected digits between 1 and 4 were presented sequentially to the subject. The subject retained the most recently displayed digit in memory while responding with the preceding digit. For example, if the first stimulus were " 1 "' and the second " 3 ," the correct response to the " 3 "' would be " 1 ." Responses were made by pressing one of four keys on the right-hand keyboard. The first response to the first stimulus of any trial was always " 1 ."

Under dual-task conditions, the digits for the classification task were presented on the left side of the display screen; the digit for the memory task was presented on the right side. The two tasks were completely independent of each other.
Digit span. In the digit-span task, the subject saw lists of numbers of different lengths. As soon as she saw a star indicating the end of the list, she repeated the numbers in the order in which they had been presented. The experimenter then indicated whether the response was correct or incorrect. Each time a list was recalled correctly, the following list had one more number. If the subject made a mistake, she was given a second chance with another list of the same length. If the subject made a second mistake, the following list had one fewer number. To get an accurate measure of the subject's memory span, the subject alternated five times between lengths that she could recall perfectly and lengths that resulted in errors.

Time estimation. For this task, the subject estimated the passage of $10 \mathrm{sec}$. To signal the beginning of the trial, the word "counting"' was displayed on the screen. When the subject thought $10 \mathrm{sec}$ had passed, she pushed the key under her left index finger and began estimating $10 \mathrm{sec}$ again immediately. She estimated six 10 -sec intervals per trial. The average estimation for the six intervals was the dependent measure. Under dual-task conditions, the word "counting" was displayed directly above the digit for the choice RT task or above the letter for the memory-search task.

Subjective workload rating scales. The multidimensional rating scale (Hauser, Childress, \& Hart, 1982) consisted of eight descriptive items that were dichotomized to extreme opposite levels: Overall Workload (Extremely High/Extremely Low), Task Difficulty (Extremely Hard/Extremely Easy), Time Pressure (Excessively Rushed/None), Performance (Perfect/Total Failure), Mental/Sensory Effort (Impossible/None), Frustration level (Totally Exasperated/Completely Fulfilled), Stress Level (Extremely Tense/Completely Relaxed), and Fatigue (Wide Awake/Exhausted).

The scales were displayed singly in the above sequence on sheets of paper. The specific scale descriptor was printed at the bottom of the scale, with the scale itself represented by a vertical line $(100 \mathrm{~mm})$ marked with lines at both ends and in the middle. The dichotomous definitions were placed adjacent to the identifying lines at the top and bottom of the scale. The subject assigned a subjective rating by drawing a line horizontally across the vertical line. The subject's ratings were computed as the distance from the bottom line $(0 \mathrm{~mm})$ to the line drawn by the subject.

\section{Procedure}

All task instructions for this experiment were either taped or written. All trials for each of the tasks were $1 \mathrm{~min}$ long; there were 45-sec breaks between trials. After each set of single- or dual-task trials, the subject used the subjective workload scales to rate the task or task combination. Three 5-min breaks were given on Day 1, and two on Days 2 and 3 .

Day 1. First, the subject completed the Jenkins Activity Survey as revised for college students (Krantz et al., 1974) and the digit-span task. She then performed 10 trials of the memory-search task at set size 5 followed by 10 trials each at set sizes 3 and 4 . After completion of the last trial with the set size of 4 , the subject rated the task using the subjective workload scales. This testing session required approximately $2 \mathrm{~h}$.

Day 2. The subject first performed 10 trials of the choice-RT task at the 3-bit level followed by 3 trials at both the 1-bit and 2-bit levels. Next she performed 6 trials each of the running-difference task and the matrix task and then 5 trials of the two tasks together. This session required approximately $1.5 \mathrm{~h}$.

Day 3. The subject began this session by reviewing the memory-search and choice-RT tasks. She performed 2 trials of the memory-search task with a set size of 5 followed by 2 trials of the choice-RT task at the 3-bit level. She then performed 2 trials of the time-estimation task. Next the subject performed 2 trials of the memory-search/time-estimation combination followed by 2 trials of the choice-RT/time-estimation combination. She then performed 6 trials each of the memory task and the classification task followed by 10 trials of this dual-task combination. This testing session required approximately $1.5 \mathrm{~h}$.

\section{RESULTS}

Because of space limitations, only the significant results will be described. 


\section{Subjective Workload Scales}

Two-way (group $\times$ task) analyses of variance (ANOVAs) were conducted on each of the 10 bipolar adjective scales. The frustration scale showed a significant group $\times$ task interaction $[F(7,98)=2.81, p=.01]$. This effect may be attributed to between-group differences in frustration under single- and dual-task conditions. Under single-task conditions, Type Bs report more frustration than Type As. However, under dual-task conditions, Type As report more frustration than Type Bs.

\section{Single-Task Performance}

A slope and intercept were calculated for each subject on the memory-search task by using the average correct RT on the ninth trial at each set size. Because the slope and the intercept of the memory-search task were significantly correlated $[\mathrm{r}=-.66, \mathrm{p}<.01]$, a multivariate analysis of variance (MANOVA) was performed on these two variables. The MANOVA revealed a significant effect of group $[F(2,13)=6.88, p=.01]$, reflecting differences in the slope. Type B subjects had a slope of about $69 \mathrm{msec}$, and Type A, $36 \mathrm{msec}$.

\section{Dual-Task Performance}

All of the data from the memory-classification, timeestimation/memory-search, and time-estimation/choiceRT task combinations were examined on a trial-by-trial basis to determine whether performance on the concurrent tasks was significantly correlated. However, in all cases, $p \geq .05$. Consequently, all data were analyzed using two-way (group $\times$ trial) ANOVAs.

Only the classification task of the memory-classification combination showed a significant effect of group $[F(1,14)$ $=5.68, \mathrm{p}=.03 \mathrm{]}$. This analysis also revealed a significant effect of trial $[\mathrm{F}(9,126)=15.64, \mathrm{p}<.01]$ and a significant group $\times$ trial interaction $[F(9,126)=2.44$, $\mathrm{p}=.01 \mathrm{~J}$. Type A subjects had faster RTs than Type B subjects $(1,950 \mathrm{msec}$ vs. $2,470 \mathrm{msec}$, respectively). However, Type B subjects improved about $1,070 \mathrm{msec}$ from the first to the last trial, versus $550 \mathrm{msec}$ for the Type A subjects.

The time-estimation task of the time-estimation/choice$\mathrm{RT}$ task combination showed a significant group $\times$ trial interaction $[F(1,14)=8.27, p=.01]$. Type A subjects became more accurate estimators with practice: They improved from 12.5 to $12.0 \mathrm{sec}$. In contrast, Type B subjects became less accurate estimators with practice: They changed from 10.9 to $13.1 \mathrm{sec}$. The choice-RT task also showed a significant effect of group $[F(1,14)=4.93, p=$ .04]. This effect reflects significantly faster RTs for the Type A subjects $(805 \mathrm{msec})$ than for the Type B subjects (954 msec).

The memory-search task of the time-estimation/memory-search combination also showed a significant main effect of group $[F(1,14)=4.57, p=.05]$. The main effect of group reflects the generally faster RTs of the Type A subjects (765 msec versus $1,000 \mathrm{msec}$ for the Type B subjects).

\section{DISCUSSION}

Under single-task conditions, the Type A subjects differed significantly from the Type B subjects on only one measure: the slope of the function relating RT to positive set size for the memory-search task. This measure reflects the speed at which items are compared in memory (Sternberg, 1964). Thus, Type A subjects make their comparisons significantly faster than Type B subjects $(36 \mathrm{msec}$ and $68 \mathrm{msec}$, respectively).

Performance differences between Type A and Type B subjects were more apparent under dual-task conditions; there were significant betweengroup differences on at least one task on three of the four dual-task combinations. Three tasks that showed significant between-group differences used RT as a dependent measure. On all three of these tasks, the RTs of the Type As were significantly faster than those of the Type Bs. With practice, the Type As estimated the passage of time more accurately than the Type Bs on the time-estimation task. These results indicate that Type A subjects perform better under multiple-task conditions than Type Bs.

There also was some evidence that the Type A/Type B distinction may account for some of the dissociations between performance and subjective estimates of workload. The Type As reported less frustration under single-task than under dual-task conditions, whereas the Type Bs reported the reverse. However, in three of the four dual-task combinations, the performance of the Type As was superior to that of the Type Bs. A similar trend was found for the fatigue scale, although it was not statistically significant $(\mathrm{p}=.06)$; Type As reported more fatigue under single-task than under dual-task conditions, whereas Type Bs reported the opposite pattern. These results support those of Manuck and Garland (1979), who found that Type As generally were less satisfied with their performance although they performed better in some conditions than Type Bs.

Thus, the Type A/Type B distinction may be helpful in explaining individual differences in multiple-task performance and the dissociations between performance and subjective estimates of workload. However, much more research is needed comparing Type A and Type B performance in multiple-task situations as a function of pacing, incentives, and changing task priorities.

\section{REFERENCES}

ACkerman, P. L., Schneider, W., \& Wickens, C. D. (1984). Deciding the existence of a time-sharing ability: A combined methodological and theoretical approach. Human Factors, 26, 71-82.

Burman, M. A., Pennebaker, J. W., \& Glass, D. C. (1975). Time consciousness, achievement striving, and the Type A coronary-prone behavior pattern. Journal of Abnormal Psychology, 84, 76-79.

DAmos, D. (1984, June). Classification schemes for individual differences in multiple-task performance and subjective estimates of workload. Paper presented at the Twentieth Annual Conference on Manual Control, San Jose, CA.

Derrick, W. L., \& WiCKens, C. D. (1984). A multiple processing resource explanation of the subjective dimensions of operator workload (Report No. EPL-84-2/ONR-84-1). Urbana, IL: University of Illinois, Engineering-Psychology Research Laboratory.

Frankenhaeuser, M., Lundberg, U., \& Forsman, L. (1980). Dissociation between sympathetic-adrenal and pituitary-adrenal responses to an achievement situation characterized by high controllability: Comparison between Type A and Type B males and females. Biological Psychology, 10, 79-91.

Friedman, M, \& Rosenman, R. H. (1974). Type A behavior and your heart. New York: Knopf.

Glass, D. C., Krakoff, L. R., Finkleman, J., Snow, B., Contrada, R., Kehoe, K., Mannucci, E., Isecke, W., Collins, C., Hilton, W., \& Elting, E. (1980). Effect of task overload upon cardiovascular and plasma catecholamine responses in Type A and B individuals. Basic and Applied Social Psychology, 1, 199-218.

Hauser, J. R., Childress, M. E., \& Hart, S. G. (1982, June). Rating consistency and component salience in subjective workload estimation. Paper presented at the Eighteenth Annual Conference on Manual Control, Dayton, OH. 
Krantz, D. S., Glass, D. C., \& SNyder, M. L. (1974). Helplessness, stress level, and the coronary-prone behavior pattern. Journal of Experimental Social Psychology, 10, 284-300.

ManUCK, S. B., \& Garland, F. N. (1979). Coronary-prone behavior pattern, task incentive, and cardiovascular reponse. Psychophysiology, 16, 136-142.

Matthews, K. A. \& Brunson, B. I. (1979). Allocation of attention and the Type A coronary-prone behavior pattern. Journal of Personality and Social Psychology, 37, 2081-2090.

STERNBERG, S. (1964). High-speed scanning in human memory. Science, 18, 953-956.

(Manuscript received for publication August 20, 1984.) 